\title{
Expansion of Rindler Coordinate Theory and Light's Doppler Effect
}

\author{
Sangwha-Yi* \\ Department of Mathematics, Taejon University, Taejon, South Korea
}

In the general theory of relativity the Rindler coordinate theory has been extended to the Rindler coordinate theory of accelerated observer that has already some initial velocity. In this paper, we present this extended theory that uses the tetrad as the new method, and discover the new inverse-coordinate transformation. Specially, if, $a_{0}<0$, this theory treats the observer with the initial velocity that does slowdown by the constant negative acceleration in the Rindler's time-space. We consider the light's Doppler Effect in the accelerated system as well as the decelerated system.

\section{Introduction}

The objective of this work is to extend the Rindler coordinate theory to the Rindler coordinate theory for accelerated observer with the initial velocity as well as for the decelerated observer.

To find the Rindler's coordinate theory, we use the following formula about the constant accelerated matter that moves on the line

$x+\frac{c^{2}}{a_{0}}=\frac{c^{2}}{a_{0}} \cosh \left(\frac{a_{0} \tau}{c}\right), t=\frac{c}{a_{0}} \sinh \left(\frac{a_{0} \tau}{c}\right)$

Here, $x$ and $t$ are spatial and temporal coordinates, respectively, in the inertial system for the constant accelerated matter. Here, $a_{0}$ is the constant acceleration, $\tau$ is the invariable time about the constant accelerated matter, $c$ is the speed of light in the inertial system in the free space-time.

In special relativity, if the matter that moves on the line is accelerated, the formula about inertial coordinate system $S(t, x, y, z)$ and $S^{\prime}\left(t^{\prime}, x^{\prime}, y^{\prime}, z^{\prime}\right)$ is

$$
V=\frac{u+v_{0}}{1+\frac{u}{c^{2}} v_{0}}, \quad V=V_{x}=\frac{d x}{d t}, u=u_{x}=\frac{d x^{\prime}}{d t^{\prime}},
$$

$$
\begin{aligned}
& d x=\frac{d x^{\prime}+v_{0} d t^{\prime}}{\sqrt{1-\frac{v_{0}^{2}}{c^{2}}}}, \quad d t=\frac{d t^{\prime}+\frac{v_{0}}{c^{2}} d x^{\prime}}{\sqrt{1-\frac{v_{0}^{2}}{c^{2}}}}, \\
& y=y^{\prime}, \quad z=z^{\prime}, \quad \frac{d y}{d t}=\frac{d y^{\prime}}{d t^{\prime}}=0, \frac{d z}{d t}=\frac{d z^{\prime}}{d t^{\prime}}=0 \\
& a=\frac{d}{d t}\left(\frac{V}{\sqrt{1-\frac{V^{2}}{c^{2}}}}\right), \quad a^{\prime}=\frac{d}{d t^{\prime}}\left(\frac{u}{\sqrt{1-\frac{u^{2}}{c^{2}}}}\right)
\end{aligned}
$$

The velocity $V$ has the initial velocity $v_{0}$ and the velocity $u$ is the velocity after acceleration $a^{\prime}$.

$$
\begin{gathered}
a=\frac{d}{d t}\left(\frac{V}{\sqrt{1-\frac{V^{2}}{c^{2}}}}\right)=\frac{\sqrt{1-\frac{v_{0}{ }^{2}}{c^{2}}}}{1+\frac{v_{0}}{c^{2}} u} \frac{d}{d t^{\prime}}\left(\frac{u+v_{0}}{\sqrt{1-\frac{v_{0}^{2}}{c^{2}}} \sqrt{1-\frac{u^{2}}{c^{2}}}}\right) \\
=\frac{1}{1+\frac{v_{0}}{c^{2}} u} \frac{d}{d t^{\prime}}\left(\frac{u+v_{0}}{\sqrt{1-\frac{u^{2}}{c^{2}}}}\right) \\
a\left(1+\frac{v_{0}}{c^{2}} u\right)=\frac{d}{d t^{\prime}}\left(\frac{u}{\sqrt{1-\frac{u^{2}}{c^{2}}}}\right)+\frac{d}{d t^{\prime}}\left(\frac{v_{0}}{\sqrt{1-\frac{u^{2}}{c^{2}}}}\right)
\end{gathered}
$$




$$
a^{\prime}=\frac{d}{d t^{\prime}}\left(\frac{u}{\sqrt{1-\frac{u^{2}}{c^{2}}}}\right), u=\frac{\int a^{\prime} d t^{\prime}}{\sqrt{1+\frac{1}{c^{2}}\left[\int a^{\prime} d t^{\prime}\right]^{2}}}
$$

Eqn. (3) is

$$
\begin{aligned}
a(1+ & \left.\frac{v_{0}}{c^{2}} u\right)=\frac{d}{d t^{\prime}}\left(\frac{u}{\sqrt{1-\frac{u^{2}}{c^{2}}}}\right)+\frac{d}{d t^{\prime}}\left(\frac{v_{0}}{\sqrt{1-\frac{u^{2}}{c^{2}}}}\right) \\
& =a^{\prime}+v_{0} \frac{d}{d t^{\prime}}\left(\sqrt{1+\frac{1}{c^{2}}\left[\int a^{\prime} d t^{\prime}\right]^{2}}\right) \\
& =a^{\prime}+v_{0} \frac{\int a^{\prime} d t^{\prime}}{\sqrt{1+\frac{1}{c^{2}}\left[\int a^{\prime} d t^{\prime}\right]^{2}}} \frac{a^{\prime}}{c^{2}} \\
& =a^{\prime}\left(1+\frac{v_{0}}{c^{2}} \frac{\int a^{\prime} d t^{\prime}}{\sqrt{1+\frac{1}{c^{2}}\left[\int a^{\prime} d t^{\prime}\right]^{2}}}\right) \\
& =a^{\prime}\left(1+\frac{v_{0}}{c^{2}} u\right)
\end{aligned}
$$

Therefore, if the matter that moves on the line is accelerated, it is $\frac{d y}{d t}=\frac{d y^{\prime}}{d t^{\prime}}=0, \frac{d z}{d t}=\frac{d z^{\prime}}{d t^{\prime}}=0$, the acceleration $a$ of the accelerated matter that has the initial velocity $v_{0}$ in the inertial coordinate system $S(t, x, y, z)$ and the other acceleration $a^{\prime}$ of the accelerated matter that does not have the initial velocity $v_{0}$ in the inertial coordinate system $S^{\prime}\left(t^{\prime}, x^{\prime}, y^{\prime}, z^{\prime}\right)$ are the same.

If the acceleration $a^{\prime}$ is the constant acceleration, $a_{0}$, the acceleration of the inertial coordinate system $S(t, x, y, z)$ and in the inertial coordinate system $S^{\prime}\left(t^{\prime}, x^{\prime}, y^{\prime}, z^{\prime}\right)$ is the constant acceleration $a_{0}$ given as

$$
a_{0}=a^{\prime}=\frac{d}{d t^{\prime}}\left(\frac{u}{\sqrt{1-\frac{u^{2}}{c^{2}}}}\right)=a=\frac{d}{d t}\left(\frac{V}{\sqrt{1-\frac{V^{2}}{c^{2}}}}\right)
$$

Therefore,

$$
V=\frac{d x}{d t}=\frac{a_{0} t+C}{\sqrt{1+\frac{1}{c^{2}}\left(a_{0} t+C\right)^{2}}},
$$

$$
\begin{aligned}
u & =\frac{d x^{\prime}}{d t^{\prime}}=\frac{a_{0} t^{\prime}}{\sqrt{1+\frac{1}{c^{2}}\left(a_{0} t^{\prime}\right)^{2}}}, \\
x^{\prime} & =\frac{c^{2}}{a_{0}}\left(\sqrt{1+\frac{1}{c^{2}}\left(a_{0} t^{\prime}\right)^{2}}-1\right) \\
& =\frac{\gamma a_{0}\left(t^{\prime}+\frac{v_{0}}{c^{2}} x^{\prime}\right)+C}{\sqrt{1+\frac{1}{c^{2}}\left(a_{0} \gamma\left(t^{\prime}+\frac{v_{0}}{c^{2}} x^{\prime}\right)+C\right)^{2}}},
\end{aligned}
$$

$C$ is the constant number

$$
=\frac{\gamma a_{0}\left(t^{\prime}+\frac{v_{0}}{c^{2}} \cdot \frac{c^{2}}{a_{0}}\left(\sqrt{1+\frac{1}{c^{2}}\left(a_{0} t^{\prime}\right)^{2}}-1\right)\right)+C}{\sqrt{1+\frac{1}{c^{2}}\left(a_{0} \gamma\left(t^{\prime}+\frac{v_{0}}{c^{2}} \cdot \frac{c^{2}}{a_{0}}\left(\sqrt{1+\frac{1}{c^{2}}\left(a_{0} t^{\prime}\right)^{2}}-1\right)\right)+C\right)^{2}}}
$$

$$
=\frac{\not a_{0} t^{\prime}+\mathcal{W}_{0} \sqrt{1+\frac{1}{c^{2}}\left(a_{0} t^{\prime}\right)^{2}}-\mathcal{W}_{0}+C}{\sqrt{1+\frac{1}{c^{2}}\left(\gamma a_{0} t^{\prime}+\mathcal{W}_{0} \sqrt{1+\frac{1}{c^{2}}\left(a_{0} t^{\prime}\right)^{2}}-\mathcal{W}_{0}+C\right)^{2}}}
$$$$
=\frac{u+v_{0}}{1+\frac{u}{c^{2}} v_{0}}=\frac{\frac{a_{0} t^{\prime}}{\sqrt{1+\frac{1}{c^{2}}\left(a_{0} t^{\prime}\right)^{2}}}+v_{0}}{1+\frac{v_{0}}{c^{2}} \frac{a_{0} t^{\prime}}{\sqrt{1+\frac{1}{c^{2}}\left(a_{0} t^{\prime}\right)^{2}}}}
$$

$$
=\frac{a_{0} t^{\prime}+v_{0} \sqrt{1+\frac{1}{c^{2}}\left(a_{0} t^{\prime}\right)^{2}}}{\sqrt{1+\frac{1}{c^{2}}\left(a_{0} t^{\prime}\right)^{2}}+\frac{v_{0}}{c^{2}} a_{0} t^{\prime}}
$$

Now,

$$
\sqrt{1+\frac{1}{c^{2}}\left(\varkappa_{0} t^{\prime}+\mathcal{W}_{0} \sqrt{1+\frac{1}{c^{2}}\left(a_{0} t^{\prime}\right)^{2}}\right)^{2}}
$$




$$
=\gamma\left(\sqrt{1+\frac{1}{c^{2}}\left(a_{0} t^{\prime}\right)^{2}}+\frac{v_{0}}{c^{2}} a_{0} t^{\prime}\right)
$$

Therefore,

$$
C=\mathcal{N}_{0}
$$

Hence,

$$
\begin{aligned}
& x=\frac{c^{2}}{a_{0}}\left(\sqrt{1+\frac{1}{c^{2}}\left(a_{0} t+\mathcal{W}_{0}\right)^{2}}-\sqrt{1+\frac{1}{c^{2}}\left(\mathcal{W}_{0}\right)^{2}}\right) \\
& =\frac{c^{2}}{a_{0}}\left(\sqrt{1+\frac{1}{c^{2}}\left(a_{0} t+\mathcal{W}_{0}\right)^{2}}-\gamma\right) \\
& =\frac{c^{2}}{a_{0}}\left(\frac{1}{\sqrt{1-\frac{V^{2}}{c^{2}}}}-\frac{1}{\sqrt{1-\frac{v_{0}^{2}}{c^{2}}}}\right) \text {, } \\
& V=\frac{a_{0} t+\mathcal{W}_{0}}{\sqrt{1+\frac{1}{c^{2}}\left(a_{0} t+\mathcal{W}_{0}\right)^{2}}} \\
& x^{\prime}=\frac{c^{2}}{a_{0}}\left(\sqrt{1+\frac{1}{c^{2}}\left(a_{0} t^{\prime}\right)^{2}}-1\right)=\frac{c^{2}}{a_{0}}\left(\frac{1}{\sqrt{1-\frac{u^{2}}{c^{2}}}}-1\right), \\
& u=\frac{a_{0} t^{\prime}}{\sqrt{1+\frac{1}{c^{2}}\left(a_{0} t^{\prime}\right)^{2}}}, \quad \gamma=\frac{1}{\sqrt{1-\frac{v_{0}^{2}}{c^{2}}}}
\end{aligned}
$$

And

$$
d \tau=\sqrt{1-V^{2} / c^{2}} d t=\frac{d t}{\sqrt{1+\frac{1}{c^{2}}\left(a_{0} t+\not \mathcal{V}_{0}\right)^{2}}},
$$$$
d \tau=\sqrt{1-u^{2} / c^{2}} d t^{\prime}=\frac{d t^{\prime}}{\sqrt{1+\frac{1}{c^{2}}\left(a_{0} t^{\prime}\right)^{2}}}
$$$$
\tau=\frac{c}{a_{0}} \sinh ^{-1}\left(\frac{a_{0}}{c} t+\gamma \frac{v_{0}}{c}\right)-\frac{c}{a_{0}} \sinh ^{-1}\left(\gamma \frac{v_{0}}{c}\right)
$$

$$
=\frac{c}{a_{0}} \sinh ^{-1}\left(\frac{a_{0}}{c} t+\gamma \frac{v_{0}}{c}\right)-\tau_{0}
$$$$
\tau+\tau_{0}=\frac{c}{a_{0}} \sinh ^{-1}\left(\frac{a_{0}}{c} t+\gamma \frac{v_{0}}{c}\right),
$$$$
\tau=\frac{c}{a_{0}} \sinh ^{-1}\left(\frac{a_{0} t^{\prime}}{c}\right)
$$

$$
\tau_{0}=\frac{c}{a_{0}} \sinh ^{-1}\left(\gamma \frac{v_{0}}{c}\right), \gamma=\frac{1}{\sqrt{1-\frac{v_{0}^{2}}{c^{2}}}}
$$

Therefore,

$$
\begin{gathered}
t+\gamma \frac{v_{0}}{a_{0}}=\frac{c}{a_{0}} \sinh \left(\frac{a_{0}}{c} \tau+\frac{a_{0}}{c} \tau_{0}\right) \\
=\frac{c}{a_{0}}\left[\sinh \left(\frac{a_{0} \tau}{c}\right) \cosh \left(\frac{a_{0} \tau_{0}}{c}\right)\right. \\
\left.+\cosh \left(\frac{a_{0} \tau}{c}\right) \sinh \left(\frac{a_{0} \tau_{0}}{c}\right)\right] \\
\gamma=\frac{1}{\sqrt{1-\frac{v_{0}^{2}}{c^{2}}}}
\end{gathered}
$$

In this time,

$$
\begin{aligned}
\tau= & \frac{c}{a_{0}} \sinh ^{-1}\left(\frac{a_{0} t^{\prime}}{c}\right) \rightarrow \sinh \left(\frac{a_{0} \tau}{c}\right)=\frac{a_{0} t^{\prime}}{c}, \\
x^{\prime}= & \frac{c^{2}}{a_{0}}\left(\sqrt{1+\frac{1}{c^{2}}\left(a_{0} t^{\prime}\right)^{2}}-1\right) \\
& \rightarrow \cosh \left(\frac{a_{0} \tau}{c}\right)=\sqrt{1+\frac{a_{0}^{2} t^{\prime 2}}{c^{2}}}=1+\frac{a_{0}}{c^{2}} x^{\prime} \\
\tau_{0}= & \frac{c}{a_{0}} \sinh ^{-1}\left(\gamma \frac{v_{0}}{c}\right) \rightarrow \sinh \left(\frac{a_{0} \tau_{0}}{c}\right)=\frac{\gamma_{0}}{c}, \\
& \cosh \left(\frac{a_{0} \tau_{0}}{c}\right)=\sqrt{1+\frac{\gamma^{2} v_{0}^{2}}{c^{2}}}=\gamma, \\
\gamma= & \frac{1}{\sqrt{1-\frac{v_{0}^{2}}{c^{2}}}}
\end{aligned}
$$


Therefore, Eqn. (12) is

$$
\begin{aligned}
& t+\gamma \frac{v_{0}}{a_{0}}=\frac{c}{a_{0}} \sinh \left(\frac{a_{0}}{c} \tau+\frac{a_{0}}{c} \tau_{0}\right) \\
& =\frac{c}{a_{0}}\left[\sinh \left(\frac{a_{0} \tau}{c}\right) \cosh \left(\frac{a_{0} \tau_{0}}{c}\right)+\cosh \left(\frac{a_{0} \tau}{c}\right) \sinh \left(\frac{a_{0} \tau_{0}}{c}\right)\right] \\
& =\frac{c}{a_{0}}\left[\gamma \sinh \left(\frac{a_{0} \tau}{c}\right)+\cosh \left(\frac{a_{0} \tau}{c}\right) \frac{\mathcal{W}_{0}}{c}\right] \\
& =\frac{c}{a_{0}}\left[\frac{a_{0} t^{\prime}}{c} \cdot \gamma+\left(1+\frac{a_{0}}{c^{2}} x^{\prime}\right) \cdot \frac{\gamma_{0}}{c}\right]=\gamma\left(t^{\prime}+\frac{v_{0}}{c^{2}} x^{\prime}\right)+\gamma \frac{v_{0}}{a_{0}}, \\
& \gamma=\frac{1}{\sqrt{1-\frac{v_{0}^{2}}{c^{2}}}}
\end{aligned}
$$

Therefore, Eqn. (10) is

$$
\begin{aligned}
& x=\frac{c^{2}}{a_{0}}\left(\sqrt{1+\frac{1}{c^{2}}\left(a_{0} t+\mathcal{W}_{0}\right)^{2}}-\gamma\right), \\
& x^{\prime}=\frac{c^{2}}{a_{0}}\left(\sqrt{1+\frac{1}{c^{2}}\left(a_{0} t^{\prime}\right)^{2}}-1\right) \\
& =\frac{c^{2}}{a_{0}}\left(\sqrt{1+\frac{1}{c^{2}}\left(a_{0} \gamma\left(t^{\prime}+\frac{v_{0}}{c^{2}} x^{\prime}\right)+\mathcal{W}_{0}\right)^{2}}-\gamma\right) \\
& =\frac{c^{2}}{a_{0}}\left(\sqrt{1+\frac{1}{c^{2}}\left(a_{0} \gamma\left(t^{\prime}+\frac{v_{0}}{c^{2}} \cdot \frac{c^{2}}{a_{0}}\left(\sqrt{1+\frac{1}{c^{2}}\left(a_{0} t^{\prime}\right)^{2}}-1\right)\right)+w_{0}\right)^{2}}-\gamma\right) \\
& =\frac{c^{2}}{a_{0}}\left(\sqrt{1+\frac{1}{c^{2}}\left(\gamma a_{0} t^{\prime}+\gamma_{0} \sqrt{1+\frac{1}{c^{2}}\left(a_{0} t^{\prime}\right)^{2}}\right)^{2}}-\gamma\right) \\
& =\frac{c^{2}}{a_{0}}\left(\sqrt{\left(\gamma \sqrt{1+\frac{1}{c^{2}}\left(a_{0} t^{\prime}\right)^{2}}+\gamma a_{0} \frac{v_{0}}{c^{2}} t^{\prime}\right)^{2}}-\gamma\right) \\
& =\gamma \frac{c^{2}}{a_{0}}\left(\sqrt{1+\frac{1}{c^{2}}\left(a_{0} t^{\prime}\right)^{2}}-1\right)+\mathcal{W}_{0} t^{\prime}=\quad \gamma\left(x^{\prime}+v_{0} t^{\prime}\right), \\
& \gamma=\frac{1}{\sqrt{1-\frac{v_{0}^{2}}{c^{2}}}}
\end{aligned}
$$

Or, by Eqns. (13) and (14)

$$
\begin{aligned}
x & =\frac{c^{2}}{a_{0}}\left(\sqrt{1+\frac{1}{c^{2}}\left(a_{0} t+\mathcal{W}_{0}\right)^{2}}-\gamma\right) \\
& =\frac{c^{2}}{a_{0}}\left(\sqrt{1+\sinh ^{2}\left(\frac{a_{0}}{c} \tau+\frac{a_{0}}{c} \tau_{0}\right)}-\gamma\right) \\
& =\frac{c^{2}}{a_{0}}\left(\cosh \left(\frac{a_{0}}{c} \tau+\frac{a_{0}}{c} \tau_{0}\right)-\gamma\right) \\
& =\frac{c^{2}}{a_{0}}\left(\cosh \left(\frac{a_{0}}{c} \tau\right) \cosh \left(\frac{a_{0}}{c} \tau_{0}\right)\right. \\
& \left.+\sinh \left(\frac{a_{0}}{c} \tau\right) \sinh \left(\frac{a_{0}}{c} \tau_{0}\right)-\gamma\right) \\
& \frac{c^{2}}{a_{0}}\left(\cosh \left(\frac{a_{0}}{c} \tau\right) \gamma+\sinh \left(\frac{a_{0}}{c} \tau\right) \frac{\mathcal{W}_{0}}{c}-\gamma\right) \\
& =\frac{c^{2}}{a_{0}} \gamma \sqrt{1+\frac{1}{c^{2}}\left(a_{0} t^{\prime}\right)^{2}}+\mathcal{W}_{0} t^{\prime}-\frac{c^{2}}{a_{0}} \gamma,
\end{aligned}
$$

$$
\gamma=\frac{1}{\sqrt{1-\frac{v_{0}^{2}}{c^{2}}}}
$$

Hence, Eqn. (1) is in the inertial coordinate system $S^{\prime}\left(t^{\prime}, x^{\prime}, y^{\prime}, z^{\prime}\right)$

$$
\begin{aligned}
& x^{\prime}=\frac{c^{2}}{a_{0}}\left(\cosh \left(\frac{a_{0} \tau}{c}\right)-1\right) \\
& t^{\prime}=\frac{c}{a_{0}} \sinh \left(\frac{a_{0} \tau}{c}\right)
\end{aligned}
$$

Therefore, in the inertial coordinate system $S(t, x, y, z)$

$$
\begin{aligned}
t & =\gamma\left(t^{\prime}+\frac{v_{0}}{c^{2}} x^{\prime}\right)=\gamma\left(\frac{c}{a_{0}} \sinh \left(\frac{a_{0}}{c} \tau\right)+\frac{v_{0}}{a_{0}}\left(\cosh \left(\frac{a_{0}}{c} \tau\right)-1\right)\right) \\
x & =\gamma\left(x^{\prime}+v_{0} t^{\prime}\right) \\
& =\gamma\left(\frac{c^{2}}{a_{0}}\left(\cosh \left(\frac{a_{0} \tau}{c}\right)-1\right)+\frac{v_{0} c}{a_{0}} \sinh \left(\frac{a_{0} \tau}{c}\right)\right),
\end{aligned}
$$

$\gamma=\frac{1}{\sqrt{1-\frac{v_{0}^{2}}{c^{2}}}}$ 
$y=y^{\prime}, \quad z=z^{\prime}$

$d t=\gamma\left(\cosh \left(\frac{a_{0}}{c} \tau\right)+\frac{v_{0}}{c} \sinh \left(\frac{a_{0}}{c} \tau\right)\right) d \tau$,

$d x=\gamma\left(c \sinh \left(\frac{a_{0}}{c} \tau\right)+v_{0} \cosh \left(\frac{a_{0}}{c} \tau\right)\right) d \tau$,

$d y=d y^{\prime}=0, d z=d z^{\prime}=0$

$V=\frac{d x}{d t}=\left(c \tanh \left(\frac{a_{0}}{c} \tau\right)+v_{0}\right) /\left(1+\frac{v_{0}}{c} \tanh \left(\frac{a_{0}}{c} \tau\right)\right)$,

$\gamma=\frac{1}{\sqrt{1-\frac{v_{0}^{2}}{c^{2}}}}$

\section{How to Use the Tetrad in the Inertial}

Coordinate System

The tetrad $e_{a}^{\mu}$ is the unit vector that is defined using the following formula

$e_{a}^{\mu} e_{b}^{v} g_{\mu v}=\eta_{a b}$

$e^{a} \mu$ is

$e^{a}{ }_{\mu}=\eta^{a b} g_{\mu v} e_{b}^{v}$

And, it is $e_{a}{ }^{\mu}$, s inverse-matrix, that is

$e^{a}{ }_{\mu} e_{b}{ }^{\mu}=\delta_{b}^{a} \quad, \quad e^{a}{ }_{\mu} e_{a}^{v}=\delta_{\mu}^{v}$

$e^{a}{ }_{\mu} e^{b}{ }_{v} \eta_{a b}=g_{\mu v}$

Here, $e^{\alpha}{ }_{\mu}(\tau)$ is the tetrad if, $\xi^{1}=\xi^{2}=\xi^{3}=0$, $d \xi^{1}=d \xi^{2}=d \xi^{3}=0$. It is not the accelerated system and it points to the fact that the accelerated motion is in line with the inertial coordinate system. Therefore, in Eqn. (22), $g_{\mu v}=\eta_{\mu v}$.
Therefore, Eqn. (22) is

$$
\begin{aligned}
& \eta_{\alpha \beta} e^{\alpha}{ }_{0}(\tau) e^{\beta}{ }_{0}(\tau)=\eta_{00}=-1 \\
& d \tau^{2}=-\frac{1}{c^{2}} \eta_{\alpha \beta} d x^{\alpha} d x^{\beta} \\
& \rightarrow-1=\eta_{\alpha \beta}\left(\frac{1}{c} \frac{d x^{\alpha}}{d \tau}\right)\left(\frac{1}{c} \frac{d x^{\beta}}{d \tau}\right)=\eta_{\alpha \beta} e^{\alpha}{ }_{0}(\tau) e^{\beta}{ }_{0}(\tau)
\end{aligned}
$$

According to Eqns. (19), (23)

$$
\begin{aligned}
& e^{\alpha}{ }_{0}(\tau)=\frac{1}{c} \frac{d x^{\alpha}}{d \tau} \\
& =\left(\gamma \cosh \left(\frac{a_{0}}{c} \tau\right)+\frac{v_{0}}{c} \gamma \sinh \left(\frac{a_{0}}{c} \tau\right), \gamma \sinh \left(\frac{a_{0}}{c} \tau\right)\right. \\
& \left.+\frac{v_{0}}{c} \gamma \cosh \left(\frac{a_{0}}{c} \tau\right), 0,0\right), \gamma=\frac{1}{\sqrt{1-\frac{v_{0}^{2}}{c^{2}}}}
\end{aligned}
$$

About $y$-axis's and $z$-axis's orientation

$e_{2}^{\alpha}(\tau)=(0,0,1,0), \quad e^{\alpha}{ }_{3}(\tau)=(0,0,0,1)$

And the other unit vector $e^{\alpha}{ }_{1}(\tau)$ has to satisfy the tetrad condition, Eqn. (22)

$$
\begin{aligned}
& e^{\alpha}{ }_{1}(\tau)=\left(\gamma \sinh \left(\frac{a_{0}}{c} \tau\right)+\frac{v_{0}}{c} \gamma \cosh \left(\frac{a_{0}}{c} \tau\right),\right. \\
& \left.\gamma \cosh \left(\frac{a_{0}}{c} \tau\right)+\frac{v_{0}}{c} \gamma \sinh \left(\frac{a_{0}}{c} \tau\right), 0,0\right), \\
& \gamma=\frac{1}{\sqrt{1-\frac{v_{0}^{2}}{c^{2}}}}
\end{aligned}
$$




\section{The Tetrad and the Expansion of the Accelerated System and the Light's Doppler Effect}

According to tetrad $e^{a}{ }_{\mu}$ in the flat Minkowski space, the inertial coordinate system $S(t, x, y, z)$ transforms the accelerated system $\xi\left(\xi^{0}, \xi^{1}, \xi^{2}, \xi^{3}\right)$. The accelerated observer of the accelerated system $\xi\left(\xi^{0}, \xi^{1}, \xi^{2}, \xi^{3}\right)$ and the accelerated matter that has the initial velocity $v_{0}$ in the inertial coordinate system $S(t, x, y, z)$ are same. Therefore, by Eqn. (22)

$$
\begin{aligned}
d \tau^{2} & =d t^{2}-\frac{1}{c^{2}}\left[d x^{2}+d y^{2}+d z^{2}\right] \\
& =-\frac{1}{c^{2}} \eta_{a b} \frac{\partial x^{a}}{\partial \xi^{\mu}} \frac{\partial x^{b}}{\partial \xi^{v}} d \xi^{\mu} d \xi^{v} \\
& =-\frac{1}{c^{2}} \eta_{a b} e^{a}{ }_{\mu} e^{b}{ }_{v} d \xi^{\mu} d \xi^{v}=-\frac{1}{c^{2}} g_{\mu v} d \xi^{\mu} d \xi^{v}
\end{aligned}
$$

$e_{\mu}^{a}=\frac{\partial x^{a}}{\partial \xi^{\mu}}$

Therefore, for saving the Rindler coordinate theory in this new method, the term $e^{\alpha}{ }_{\mu}\left(\xi^{0}\right)$ is used by Eqns. (24), (25) and (26), where $\xi^{0}$ is used instead of $\tau$. Here, $d y=d \xi^{2} \neq 0$ and $d z=d \xi^{3} \neq 0$, because it is the matter that the accelerated observer of the accelerated system $\xi\left(\xi^{0}, \xi^{1}, \xi^{2}, \xi^{3}\right)$ observes.

The unit vector $e^{\alpha}{ }_{1}\left(\xi^{0}\right)$ is

$$
\begin{array}{r}
e_{1}^{\alpha}\left(\xi^{0}\right)=\frac{\partial x^{\alpha}}{\partial \xi^{1}}=\left(\gamma \sinh \left(\frac{a_{0}}{c} \xi^{0}\right)\right. \\
+\frac{v_{0}}{c} \gamma \cosh \left(\frac{a_{0}}{c} \xi^{0}\right),
\end{array}
$$$$
\left.\gamma \cosh \left(\frac{a_{0}}{c} \xi^{0}\right)+\frac{v_{0}}{c} \gamma \sinh \left(\frac{a_{0}}{c} \xi^{0}\right), 0,0\right),
$$

$$
\gamma=\frac{1}{\sqrt{1-\frac{v_{0}^{2}}{c^{2}}}}
$$

$$
\frac{\partial e^{\alpha}{ }_{1}\left(\xi^{0}\right)}{c \partial \xi^{0}}=\frac{\partial^{2} x^{\alpha}}{\partial \xi^{1} c \partial \xi^{0}}=\frac{\partial e^{\alpha}{ }_{0}\left(\xi^{0}\right)}{\partial \xi^{1}}
$$

Therefore, the vector $e^{\alpha}{ }_{0}\left(\xi^{0}\right)$ is

$$
\begin{gathered}
e^{\alpha}{ }_{0}\left(\xi^{0}\right)=\frac{\partial x^{\alpha}}{c \partial \xi^{0}}=\left(( 1 + \frac { a _ { 0 } } { c ^ { 2 } } \xi ^ { 1 } ) \left(\gamma \cosh \left(\frac{a_{0}}{c} \xi^{0}\right)\right.\right. \\
\left.+\frac{v_{0}}{c} \gamma \sinh \left(\frac{a_{0}}{c} \xi^{0}\right)\right),\left(1+\frac{a_{0}}{c^{2}} \xi^{1}\right)\left(\gamma \sinh \left(\frac{a_{0}}{c} \xi^{0}\right)\right. \\
\left.\left.+\frac{v_{0}}{c} \gamma \cosh \left(\frac{a_{0}}{c} \xi^{0}\right)\right), 0,0\right), \gamma=\frac{1}{\sqrt{1-\frac{v_{0}{ }^{2}}{c^{2}}}}
\end{gathered}
$$

About $y$-axis's and $z$-axis's orientation, the unit vector $e^{\alpha}{ }_{2}\left(\xi^{0}\right)$ and $e^{\alpha}{ }_{3}\left(\xi^{0}\right)$ is

$e_{2}^{\alpha}\left(\xi^{0}\right)=\frac{\partial x^{\alpha}}{\partial \xi^{2}}=(0,0,1,0)$,

$e^{\alpha}{ }_{3}\left(\xi^{0}\right)=\frac{\partial x^{\alpha}}{\partial \xi^{3}}=(0,0,0,1)$

The differential coordinate transformation is

$$
\begin{aligned}
d x^{\alpha}= & \frac{\partial x^{\alpha}}{\partial \xi^{\mu}} d \xi^{\mu}=\frac{\partial x^{\alpha}}{c \partial \xi^{0}} c d \xi^{0} \\
& +\frac{\partial x^{\alpha}}{\partial \xi^{1}} d \xi^{1}+\frac{\partial x^{\alpha}}{\partial \xi^{2}} d \xi^{2}+\frac{\partial x^{\alpha}}{\partial \xi^{3}} d \xi^{3} \\
= & e^{\alpha}{ }_{0}\left(\xi^{0}\right) c d \xi^{0}+e^{\alpha}{ }_{1}\left(\xi^{0}\right) d \xi^{1} \\
& +e^{\alpha}{ }_{2}\left(\xi^{0}\right) d \xi^{2}+e^{\alpha}{ }_{3}\left(\xi^{0}\right) d \xi^{3}
\end{aligned}
$$

$c d t=\gamma\left[\left(1+\frac{a_{0}}{c^{2}} \xi^{1}\right)\left\{\cosh \left(\frac{a_{0} \xi^{0}}{c}\right)\right.\right.$ 


$$
\begin{aligned}
& \left.+\frac{v_{0}}{c} \sinh \left(\frac{a_{0} \xi^{0}}{c}\right)\right\} c d \xi^{0} \\
& \left.+\left\{\sinh \left(\frac{a_{0} \xi^{0}}{c}\right)+\frac{v_{0}}{c} \cosh \left(\frac{a_{0} \xi^{0}}{c}\right)\right\} d \xi^{1}\right]
\end{aligned}
$$

$d x=\gamma\left[\left(1+\frac{a_{0}}{c^{2}} \xi^{1}\right)\left\{\sinh \left(\frac{a_{0} \xi^{0}}{c}\right)\right.\right.$

$$
\left.+\frac{v_{0}}{c} \cosh \left(\frac{a_{0} \xi^{0}}{c}\right)\right\} c d \xi^{0}
$$$$
\left.+\left\{\cosh \left(\frac{a_{0} \xi^{0}}{c}\right)+\frac{v_{0}}{c} \sinh \left(\frac{a_{0} \xi^{0}}{c}\right)\right\} d \xi^{1}\right],
$$

$$
\gamma=\frac{1}{\sqrt{1-\frac{v_{0}^{2}}{c^{2}}}}
$$

$$
d y=d \xi^{2}, d z=d \xi^{3}
$$

Therefore, if Eqns. (33), (34) and (35) integrate, then the Rindler coordinate theory's coordinate transformation of the accelerated observer with the initial velocity is finally found.

$$
\begin{aligned}
c t= & \gamma\left(\frac{c^{2}}{a_{0}}+\xi^{1}\right)\left\{\sinh \left(\frac{a_{0} \xi^{0}}{c}\right)\right. \\
& \left.+\frac{v_{0}}{c} \cosh \left(\frac{a_{0} \xi^{0}}{c}\right)\right\}-\gamma \frac{v_{0} c}{a_{0}} \\
x= & \gamma\left(\frac{c^{2}}{a_{0}}+\xi^{1}\right)\left\{\cosh \left(\frac{a_{0} \xi^{0}}{c}\right)\right. \\
& \left.+\frac{v_{0}}{c} \sinh \left(\frac{a_{0} \xi^{0}}{c}\right)\right\}-\gamma \frac{c^{2}}{a_{0}} \\
y= & \xi^{2}, z=\xi^{3}, \gamma=\frac{1}{\sqrt{1-\frac{v_{0}{ }^{2}}{c^{2}}}}
\end{aligned}
$$

Therefore, the new inverse-coordinate transformation of the Rindler coordinate theory of the accelerated observer with the initial velocity is

$$
\begin{gathered}
\frac{\left(c t+\gamma \frac{v_{0} c}{a_{0}}\right)}{\left(x+\gamma \frac{c^{2}}{a_{0}}\right)}=\frac{\tanh \left(\frac{a_{0} \xi^{0}}{c}\right)+\frac{v_{0}}{c}}{1+\frac{v_{0}}{c} \cdot \tanh \left(\frac{a_{0} \xi^{0}}{c}\right)} \\
\xi^{0}=\frac{c}{a_{0}} \tanh ^{-1}\left[\frac{\left(c t+\gamma \frac{v_{0} c}{a_{0}}\right)}{\left(x+\gamma \frac{c^{2}}{a_{0}}\right)}-\frac{v_{0}}{c}\right. \\
1-\frac{v_{0}}{c} \cdot \frac{\left(c t+\gamma \frac{v_{0} c}{a_{0}}\right)}{\left(x+\gamma \frac{c^{2}}{a_{0}}\right)}
\end{gathered}
$$$$
\gamma=\frac{1}{\sqrt{1-\frac{v_{0}^{2}}{c^{2}}}}
$$

$$
\begin{aligned}
& \left(x+\gamma \frac{c^{2}}{a_{0}}\right)^{2}-\left(c t+\gamma \frac{v_{0} c}{a_{0}}\right)^{2} \\
& =\left(\frac{c^{2}}{a_{0}}+\xi^{1}\right)^{2} \gamma^{2}\left(1-\frac{v_{0}^{2}}{c^{2}}\right)=\left(\frac{c^{2}}{a_{0}}+\xi^{1}\right)^{2} \\
& \xi^{1}=\sqrt{\left(x+\gamma \frac{c^{2}}{a_{0}}\right)^{2}-\left(c t+\gamma \frac{v_{0} c}{a_{0}}\right)^{2}}-\frac{c^{2}}{a_{0}} \\
& \xi^{2}=y, \xi^{3}=z, \gamma=\frac{1}{\sqrt{1-\frac{v_{0}^{2}}{c^{2}}}}
\end{aligned}
$$

Therefore, the invariable time $d \tau$ of the Rindler coordinate theory of the accelerated observer with the initial velocity is by Eqns. (33), (34), (35)

$$
d \tau^{2}=d t^{2}-\frac{1}{c^{2}}\left[d x^{2}+d y^{2}+d z^{2}\right]
$$


$=\left(1+\frac{a_{0}}{c^{2}} \xi^{1}\right)^{2}\left(d \xi^{0}\right)^{2}-\frac{1}{c^{2}}\left[\left(d \xi^{1}\right)^{2}+\left(d \xi^{2}\right)^{2}+\left(d \xi^{3}\right)^{2}\right]$

Hence, the invariable time $d \tau$ of the new accelerated system theory of the accelerated observer that has the initial velocity $v_{0}$ is not related to the initial velocity $v_{0}$.

Hence, Riemann curvature tensor $R_{\mu \nu \rho}^{\lambda}(x), R_{\alpha \beta \gamma}^{\delta}(\xi)$ is

$g_{00}=-\left(1+\frac{a_{0}}{c^{2}} \xi^{1}\right)^{2}, g_{11}=g_{22}=g_{33}=1$,

$g^{00}=-1 /\left(1+\frac{a_{0}}{c^{2}} \xi^{1}\right)^{2}, g^{11}=g^{22}=g^{33}=1$

$\Gamma^{1}{ }_{00}=\frac{1}{2} g^{11}\left(\frac{\partial g_{00}}{\partial \xi^{1}}\right)=\frac{1}{2} \cdot-2\left(1+\frac{a_{0}}{c^{2}} \xi^{1}\right) \frac{a_{0}}{c^{2}}$

$$
=-\left(1+\frac{a_{0}}{c^{2}} \xi^{1}\right) \frac{a_{0}}{c^{2}}
$$

$\Gamma_{10}^{0}=\Gamma_{01}^{0}=\frac{1}{2} g^{00}\left(\frac{\partial g_{00}}{\partial \xi^{1}}\right)=\frac{1}{2} \cdot-1 /\left(1+\frac{a_{0}}{c^{2}} \xi^{1}\right)^{2}$.

$$
-2\left(1+\frac{a_{0}}{c^{2}} \xi^{1}\right) \frac{a_{0}}{c^{2}}=\frac{1}{\left(1+\frac{a_{0}}{c^{2}} \xi^{1}\right)} \frac{a_{0}}{c^{2}}
$$

$R_{\alpha \beta \gamma}^{\delta_{\alpha \beta}}(\xi)=\frac{\partial \Gamma_{\alpha \beta}^{\delta}}{\partial \xi^{\gamma}}-\frac{\partial \Gamma_{\alpha \gamma}^{\delta}}{\partial \xi^{\beta}}+\Gamma_{\alpha \beta}^{\sigma} \Gamma_{\sigma \gamma}^{\delta}-\Gamma_{\alpha \gamma}^{\sigma} \Gamma_{\sigma \beta}^{\delta}$

$R_{001}^{1}(\xi)=-R_{010}^{1}(\xi)=\frac{\partial \Gamma^{1} 00}{\partial \xi^{1}}-\Gamma_{01}^{0} \Gamma_{00}^{1}$

$$
=-\frac{a_{0}^{2}}{c^{4}}+\frac{a_{0}^{2}}{c^{4}}=0
$$

Otherwise, $R_{\alpha \beta \gamma}^{\delta}(\xi)=0$

$0=R_{\mu v \rho}^{\lambda}(c t, x, y, z)$

$$
=\frac{\partial x^{\lambda}}{\partial \xi^{\delta}} \frac{\partial \xi^{\alpha}}{\partial x^{\mu}} \frac{\partial \xi^{\beta}}{\partial x^{v}} \frac{\partial \xi^{\gamma}}{\partial x^{\rho}} R_{\alpha \beta \gamma}^{\delta}\left(c \xi^{0}, \xi^{1}, \xi^{2}, \xi^{3}\right),
$$

$0=R_{\alpha \beta \gamma}^{\delta}\left(c \xi^{0}, \xi^{1}, \xi^{2}, \xi^{3}\right)$
Therefore, the accelerated observer with the initial velocity is in the flat Minkowski space.

About $x$-axis's light speed,

$$
\begin{aligned}
& d y=d \xi^{2}=d z=d \xi^{3}=0, y=\xi^{2}=z=\xi^{3}=0 \\
& c d t=d x \quad, \quad c t=x \\
& c d \xi^{0}=\frac{d \xi^{1}}{\left(1+\frac{a_{0}}{c^{2}} \xi^{1}\right)} \\
& c \xi^{0}=\frac{c^{2}}{a_{0}} \ln \left|1+\frac{a_{0}}{c^{2}} \xi^{1}\right| \rightarrow\left(1+\frac{a_{0}}{c^{2}} \xi^{1}\right)=e^{\frac{a_{0} \xi^{0}}{c}} \\
& \rightarrow\left(\frac{c^{2}}{a_{0}}+\xi^{1}\right)=\frac{c^{2}}{a_{0}} e^{\frac{a_{0}}{c} \xi^{0}}
\end{aligned}
$$

In this time, if we use the accelerated system coordinate transformation, then Eqn. (36), Eqn. (37)

$$
\begin{aligned}
& c t=\gamma\left(\frac{c^{2}}{a_{0}}+\xi^{1}\right)\left\{\sinh \left(\frac{a_{0} \xi^{0}}{c}\right)+\frac{v_{0}}{c} \cosh \left(\frac{a_{0} \xi^{0}}{c}\right)\right\}-\gamma \frac{v_{0} c}{a_{0}} \\
& =\gamma \frac{c^{2}}{a_{0}} e^{\frac{a_{0}}{c} \xi^{0}}\left\{\frac{e^{\frac{a_{0}}{c} \xi^{0}}-e^{-\frac{a_{0}}{c} \xi^{0}}}{2}+\frac{v_{0}}{c} \frac{e^{\frac{a_{0}}{c} \xi^{0}}+e^{-\frac{a_{0}}{c} \xi^{0}}}{2}\right\}-\gamma \frac{v_{0} c}{a_{0}}
\end{aligned}
$$$$
=\gamma \frac{c^{2}}{a_{0}}\left\{\frac{e^{2 \frac{a_{0}}{c} \xi^{0}}-1}{2}+\frac{v_{0}}{c}\left(\frac{e^{2 \frac{a_{0}}{c} \xi^{0}}+1}{2}-1\right)\right\}
$$$$
=\gamma \frac{c^{2}}{a_{0}}\left\{\frac{e^{2 \frac{a_{0}}{c} \xi^{0}}-1}{2}+\frac{v_{0}}{c} \frac{e^{2 \frac{a_{0}}{c} \xi^{0}}-1}{2}\right\}
$$$$
=x=\gamma\left(\frac{c^{2}}{a_{0}}+\xi^{1}\right)\left\{\cosh \left(\frac{a_{0} \xi^{0}}{c}\right)+\frac{v_{0}}{c} \sinh \left(\frac{a_{0} \xi^{0}}{c}\right)\right\}-\gamma \frac{c^{2}}{a_{0}}
$$

$=\gamma \frac{c^{2}}{a_{0}} e^{\frac{a_{0}}{c} \xi^{0}}\left\{\frac{e^{\frac{a_{0}}{c} \xi^{0}}+e^{-\frac{a_{0}}{c} \xi^{0}}}{2}+\frac{v_{0}}{c} \frac{e^{\frac{a_{0}}{c^{0}}}-e^{-\frac{a_{0}}{c} \xi^{0}}}{2}\right\}-\gamma \frac{c^{2}}{a_{0}}$

$=\gamma \frac{c^{2}}{a_{0}}\left\{\left(\frac{e^{2 \frac{a_{0}}{c} \xi^{0}}+1}{2}-1\right)+\frac{v_{0}}{c} \frac{e^{2 \frac{a_{0}}{c} \xi^{0}}-1}{2}\right\}$ 


$$
=\gamma \frac{c^{2}}{a_{0}}\left\{\frac{e^{2 \frac{a_{0}}{c} \xi^{0}}-1}{2}+\frac{v_{0}}{c} \frac{e^{2 \frac{a_{0}}{c} \xi^{0}}-1}{2}\right\}
$$

According to Eqn. (45), the Doppler effect of the accelerated system with initial velocity and the inertial system is

$$
\begin{aligned}
c t & =\gamma \frac{c^{2}}{a_{0}}\left\{\frac{e^{2 \frac{a_{0}}{c} \xi^{0}}-1}{2}+\frac{v_{0}}{c} \frac{e^{2 \frac{a_{0}}{c} \xi^{0}}-1}{2}\right\} \\
& =\frac{c^{2}}{a_{0}} \frac{1+\frac{v_{0}}{c}}{\sqrt{1-v_{0}^{2} / c^{2}}} \frac{e^{2 \frac{a_{0}}{c} \xi^{0}}-1}{2}
\end{aligned}
$$

$$
\begin{aligned}
& c\left(t_{2}-t_{1}\right)=\frac{c}{v}=\frac{c^{2}}{a_{0}} \frac{1+\frac{v_{0}}{c}}{\sqrt{1-v_{0}^{2} / c^{2}}} \frac{e^{2 \frac{a_{0}}{c} \xi_{2}^{0}}-e^{2 \frac{a_{0}}{c} \xi_{1}^{0}}}{2}, \\
& e^{x} \approx 1+x+\frac{x^{2}}{2} \approx \frac{c^{2}}{a_{0}} \frac{\sqrt{1+v_{0} / c}}{\sqrt{1-v_{0} / c}}\left\{\frac{a_{0}}{c}\left(\xi_{2}{ }^{0}-\xi_{1}{ }^{0}\right)\right. \\
& \left.+\frac{a_{0}{ }^{2}}{c^{2}}\left(\xi_{2}^{0}+\xi_{1}^{0}\right)\left(\xi_{2}^{0}-\xi_{1}^{0}\right)\right\} \\
& =\frac{\sqrt{1+v_{0} / c}}{\sqrt{1-v_{0} / c}}\left\{1+\frac{a_{0}}{c}\left(\xi_{1}^{0}+\xi_{2}{ }^{0}\right)\right\} \frac{c}{v_{\xi}} \\
& v \approx \frac{\sqrt{1-v_{0} / c}}{\sqrt{1+v_{0} / c}} \frac{v_{\xi}}{\left\{1+\frac{a_{0}}{c}\left(\xi_{1}{ }^{0}+\xi_{2}{ }^{0}\right)\right\}}>0 \\
& v=\frac{1}{t_{2}-t_{1}}, v_{\xi}=\frac{1}{\xi_{2}^{0}-\xi_{1}^{0}}
\end{aligned}
$$

\section{The Decelerated System and the Light's \\ Doppler Effect}

Specially, if, $a_{0}<0$, this theory shows that the observer with the initial velocity does slowdown by the constant negative acceleration in the Rindler's time-space. This system can be called the decelerated system in the Rindler's space-time. Therefore, if, $a_{0}>0$, it uses $-a_{0}$ instead of $a_{0}$, in Eqn. (29), Eqn. (31), in the decelerated system,

$$
\begin{aligned}
d \tau^{2} & =d t^{2}-\frac{1}{c^{2}}\left[d x^{2}+d y^{2}+d z^{2}\right] \\
& =-\frac{1}{c^{2}} \eta_{a b} \frac{\partial x^{a}}{\partial \xi^{\mu}} \frac{\partial x^{b}}{\partial \xi^{v}} d \xi^{\mu} d \xi^{v} \\
& =-\frac{1}{c^{2}} \eta_{a b} e^{a}{ }_{\mu} e^{b}{ }_{\nu} d \xi^{\mu} d \xi^{v}=-\frac{1}{c^{2}} g_{\mu \nu} d \xi^{\mu} d \xi^{v} \\
e^{a}{ }_{\mu} & =\frac{\partial x^{a}}{\partial \xi^{\mu}}
\end{aligned}
$$

Therefore, the unit vector $e^{\alpha}{ }_{1}\left(\xi^{0}\right)$ is

$$
\begin{aligned}
e^{\alpha}{ }_{1}\left(\xi^{0}\right)=\frac{\partial x^{\alpha}}{\partial \xi^{1}} & =\left(-\gamma \sinh \left(\frac{a_{0}}{c} \xi^{0}\right)\right. \\
& +\frac{v_{0}}{c} \gamma \cosh \left(\frac{a_{0}}{c} \xi^{0}\right),
\end{aligned}
$$$$
\left.\gamma \cosh \left(\frac{a_{0}}{c} \xi^{0}\right)-\frac{v_{0}}{c} \gamma \sinh \left(\frac{a_{0}}{c} \xi^{0}\right), 0,0\right)
$$$$
\gamma=\frac{1}{\sqrt{1-\frac{v_{0}^{2}}{c^{2}}}}
$$

$$
\frac{\partial e^{\alpha}{ }_{1}\left(\xi^{0}\right)}{c \partial \xi^{0}}=\frac{\partial^{2} x^{\alpha}}{\partial \xi^{1} c \partial \xi^{0}}=\frac{\partial e^{\alpha}{ }_{0}\left(\xi^{0}\right)}{\partial \xi^{1}}
$$

Therefore, the vector $e^{\alpha}{ }_{0}\left(\xi^{0}\right)$ is

$$
\begin{aligned}
& e_{0}^{\alpha}\left(\xi^{0}\right)=\frac{\partial x^{\alpha}}{c \partial \xi^{0}} \\
& =\left(\left(1-\frac{a_{0}}{c^{2}} \xi^{1}\right)\left(\gamma \cosh \left(\frac{a_{0}}{c} \xi^{0}\right)-\frac{v_{0}}{c} \gamma \sinh \left(\frac{a_{0}}{c} \xi^{0}\right)\right),\right. \\
& \left(1-\frac{a_{0}}{c^{2}} \xi^{1}\right)\left(-\gamma \sinh \left(\frac{a_{0}}{c} \xi^{0}\right)\right.
\end{aligned}
$$




$$
\left.\left.+\frac{v_{0}}{c} \gamma \cosh \left(\frac{a_{0}}{c} \xi^{0}\right)\right), 0,0\right), \gamma=\frac{1}{\sqrt{1-\frac{v_{0}{ }^{2}}{c^{2}}}}
$$

About the orientation of $y$-axis and $z$-axis, unit vectors $e^{\alpha}{ }_{2}\left(\xi^{0}\right)$ and $e^{\alpha}{ }_{3}\left(\xi^{0}\right)$ are

$$
\begin{aligned}
& e_{2}^{\alpha}\left(\xi^{0}\right)=\frac{\partial x^{\alpha}}{\partial \xi^{2}}=(0,0,1,0), \\
& e_{3}^{\alpha}\left(\xi^{0}\right)=\frac{\partial x^{\alpha}}{\partial \xi^{3}}=(0,0,0,1)
\end{aligned}
$$

In the decelerated system, the differential coordinate transformation is

$$
\begin{aligned}
d x^{\alpha}= & \frac{\partial x^{\alpha}}{\partial \xi^{\mu}} d \xi^{\mu}=\frac{\partial x^{\alpha}}{c \partial \xi^{0}} c d \xi^{0}+\frac{\partial x^{\alpha}}{\partial \xi^{1}} d \xi^{1} \\
& +\frac{\partial x^{\alpha}}{\partial \xi^{2}} d \xi^{2}+\frac{\partial x^{\alpha}}{\partial \xi^{3}} d \xi^{3} \\
= & e^{\alpha}{ }_{0}\left(\xi^{0}\right) c d \xi^{0}+e^{\alpha}{ }_{1}\left(\xi^{0}\right) d \xi^{1} \\
& +e^{\alpha}{ }_{2}\left(\xi^{0}\right) d \xi^{2}+e^{\alpha}{ }_{3}\left(\xi^{0}\right) d \xi^{3} \\
c d t= & \gamma\left[( 1 - \frac { a _ { 0 } } { c ^ { 2 } } \xi ^ { 1 } ) \left\{\cosh \left(\frac{a_{0} \xi^{0}}{c}\right)\right.\right. \\
& \left.-\frac{v_{0}}{c} \sinh \left(\frac{a_{0} \xi^{0}}{c}\right)\right\} c d \xi^{0} \\
& \left.+\left\{-\sinh \left(\frac{a_{0} \xi^{0}}{c}\right)+\frac{v_{0}}{c} \cosh \left(\frac{a_{0} \xi^{0}}{c}\right)\right\} d \xi^{1}\right] \\
& \left.+\left\{\cosh \left(\frac{a_{0} \xi^{0}}{c}\right)-\frac{v_{0}}{c} \sinh \left(\frac{a_{0} \xi^{0}}{c}\right)\right\} d \xi^{1}\right], \\
d x= & \gamma\left[( 1 - \frac { a _ { 0 } } { c ^ { 2 } } \xi ^ { 1 } ) \left\{-\sinh \left(\frac{a_{0} \xi^{0}}{c}\right)\right.\right. \\
& \left.+\frac{v_{0}}{c} \cosh \left(\frac{a_{0} \xi^{0}}{c}\right)\right\} c d \xi^{0} \\
&
\end{aligned}
$$

$$
\gamma=\frac{1}{\sqrt{1-\frac{v_{0}^{2}}{c^{2}}}}
$$

$$
d y=d \xi^{2}, d z=d \xi^{3}
$$

Therefore, if, $a_{0}>0$, the invariable time $d \tau$ of the Rindler coordinate theory of the decelerated observer is given by Eqns. (55), (56), (57)

$$
\begin{aligned}
& d \tau^{2}=d t^{2}-\frac{1}{c^{2}}\left[d x^{2}+d y^{2}+d z^{2}\right] \\
& =\left(1-\frac{a_{0}}{c^{2}} \xi^{1}\right)^{2}\left(d \xi^{0}\right)^{2}-\frac{1}{c^{2}}\left[\left(d \xi^{1}\right)^{2}+\left(d \xi^{2}\right)^{2}+\left(d \xi^{3}\right)^{2}\right]
\end{aligned}
$$

If, $a_{0}>0$, in the decelerated system the Riemann curvature tensor, $R_{\mu v \rho}^{\lambda}(x), R_{\alpha \beta \gamma}^{\delta}(\xi)$, is

$$
\begin{aligned}
& g_{00}=-\left(1-\frac{a_{0}}{c^{2}} \xi^{1}\right)^{2}, g_{11}=g_{22}=g_{33}=1 \\
& g^{00}=-1 /\left(1-\frac{a_{0}}{c^{2}} \xi^{1}\right)^{2}, g^{11}=g^{22}=g^{33}=1 \\
& \Gamma_{00}^{1}=\frac{1}{2} g^{11}\left(\frac{\partial g_{00}}{\partial \xi^{1}}\right)=\frac{1}{2} \cdot-2\left(1-\frac{a_{0}}{c^{2}} \xi^{1}\right) \\
& \quad-\frac{a_{0}}{c^{2}}=\left(1-\frac{a_{0}}{c^{2}} \xi^{1}\right) \frac{a_{0}}{c^{2}} \\
& \Gamma_{10}^{0}=\Gamma_{01}^{0}=\frac{1}{2} g^{00}\left(\frac{\partial g_{00}}{\partial \xi^{1}}\right)=\frac{1}{2} \cdot-1 /\left(1-\frac{a_{0}}{c^{2}} \xi^{1}\right)^{2} . \\
& -2\left(1-\frac{a_{0}}{c^{2}} \xi^{1}\right) \cdot-\frac{a_{0}}{c^{2}}=-\frac{1}{\left(1-\frac{a_{0}}{c^{2}} \xi^{1}\right)} \frac{a_{0}}{c^{2}} \\
& R_{\alpha \beta \gamma}^{\delta}(\xi)=\frac{\partial \Gamma_{\alpha \beta}^{\delta}}{\partial \xi^{\gamma}}-\frac{\partial \Gamma_{\alpha \gamma}^{\delta}}{\partial \xi^{\beta}}+\Gamma_{\alpha \beta}^{\sigma} \Gamma_{\sigma \gamma}^{\delta}-\Gamma_{\alpha \gamma}^{\sigma} \Gamma_{\sigma \beta}^{\delta} \\
& R_{001}^{1}(\xi)=-R_{010}^{1}(\xi)=\frac{\partial \Gamma_{00}^{1}}{\partial \xi^{1}}
\end{aligned}
$$




$$
-\Gamma_{01}^{0} \Gamma_{00}^{1}=-\frac{a_{0}^{2}}{c^{4}}+\frac{a_{0}^{2}}{c^{4}}=0,
$$

Otherwise, $R_{\alpha \beta \gamma}^{\delta}(\xi)=0$

$0=R_{\mu v \rho}^{\lambda}(c t, x, y, z)$

$=\frac{\partial x^{\lambda}}{\partial \xi^{\delta}} \frac{\partial \xi^{\alpha}}{\partial x^{\mu}} \frac{\partial \xi^{\beta}}{\partial x^{v}} \frac{\partial \xi^{\gamma}}{\partial x^{\rho}} R_{\alpha \beta \gamma}^{\delta}\left(c \xi^{0}, \xi^{1}, \xi^{2}, \xi^{3}\right)$,

$0=R_{\alpha \beta \gamma}^{\delta}\left(c \xi^{0}, \xi^{1}, \xi^{2}, \xi^{3}\right)$

Therefore, the decelerated system is in the flat Minkowski space.

Therefore, if, $a_{0}>0$, in Eqn. (36) and Eqn. (37), and if we use $-a_{0}$ instead of $a_{0}$ in the decelerated system, then the coordinate transformation is

$$
\begin{aligned}
& c t=\gamma\left(\frac{c^{2}}{-a_{0}}+\xi^{1}\right)\left\{\sinh \left(\frac{-a_{0} \xi^{0}}{c}\right)\right. \\
& \left.+\frac{v_{0}}{c} \cosh \left(\frac{-a_{0} \xi^{0}}{c}\right)\right\}-\gamma \frac{v_{0} c}{-a_{0}} \\
& =\gamma\left(\frac{c^{2}}{a_{0}}-\xi^{1}\right)\left\{\sinh \left(\frac{a_{0} \xi^{0}}{c}\right)-\frac{v_{0}}{c} \cosh \left(\frac{a_{0} \xi^{0}}{c}\right)\right\}+\gamma \frac{v_{0} c}{a_{0}}
\end{aligned}
$$

$$
\begin{aligned}
& x=\gamma\left(\frac{c^{2}}{-a_{0}}+\xi^{1}\right)\left\{\cosh \left(\frac{-a_{0} \xi^{0}}{c}\right)\right. \\
& \left.+\frac{v_{0}}{c} \sinh \left(\frac{-a_{0} \xi^{0}}{c}\right)\right\}-\gamma \frac{c^{2}}{-a_{0}} \\
& =-\gamma\left(\frac{c^{2}}{a_{0}}-\xi^{1}\right)\left\{\cosh \left(\frac{a_{0} \xi^{0}}{c}\right)-\frac{v_{0}}{c} \sinh \left(\frac{a_{0} \xi^{0}}{c}\right)\right\}+\gamma \frac{c^{2}}{a_{0}}
\end{aligned}
$$

$$
y=\xi^{2}, z=\xi^{3}, \gamma=\frac{1}{\sqrt{1-\frac{v_{0}^{2}}{c^{2}}}}
$$

If, $a_{0}>0$, in Eqn. (39) and Eqn. (40), and if we use $-a$ instead of $a_{0}$, in the decelerated system, then the inverse-coordinate transformation is

$$
\begin{aligned}
& \frac{\left(c t-\gamma \frac{v_{0} c}{a_{0}}\right)}{\left(x-\gamma \frac{c^{2}}{a_{0}}\right)}=\frac{\tanh \left(-\frac{a_{0} \xi^{0}}{c}\right)+\frac{v_{0}}{c}}{1+\frac{v_{0}}{c} \cdot \tanh \left(-\frac{a_{0} \xi^{0}}{c}\right)} \\
& \xi^{0}=\frac{c}{-a_{0}} \tanh ^{-1}\left[\frac{\frac{\left(c t+\gamma \frac{v_{0} c}{-a_{0}}\right)}{\left(x+\gamma \frac{c^{2}}{-a_{0}}\right)}-\frac{v_{0}}{c}}{1-\frac{v_{0}}{c} \cdot \frac{\left(c t+\gamma \frac{v_{0} c}{-a_{0}}\right)}{\left(x+\gamma \frac{c^{2}}{-a_{0}}\right)}}\right] \\
& \left.-\frac{\left(c t-\gamma \frac{v_{0} c}{a_{0}}\right)}{1-\frac{v_{0}}{c}}\right]_{\left.\frac{c^{2}}{a_{0}}\right)}^{-\frac{v_{0}}{c} \cdot \frac{\left(c t-\gamma \frac{v_{0} c}{a_{0}}\right)}{\left(x-\gamma \frac{c^{2}}{a_{0}}\right)}} \\
& \left(x-\gamma \frac{c^{2}}{a_{0}}\right)^{2}-\left(c t-\gamma \frac{v_{0} c}{a_{0}}\right)^{2} \\
& =\left(-\frac{c^{2}}{a_{0}}+\xi^{1}\right)^{2} \gamma^{2}\left(1-\frac{v_{0}^{2}}{c^{2}}\right)=\left(-\frac{c^{2}}{a_{0}}+\xi^{1}\right)^{2}
\end{aligned}
$$

Specially, if, $x=0, c t=0, v_{0}=0$, it has to be $\xi^{1}=0$. Therefore,

$$
\begin{aligned}
\xi^{1} & =-\sqrt{\left(x+\gamma \frac{c^{2}}{-a_{0}}\right)^{2}-\left(c t+\gamma \frac{v_{0} c}{-a_{0}}\right)^{2}}-\frac{c^{2}}{-a_{0}} \\
& =-\sqrt{\left(x-\gamma \frac{c^{2}}{a_{0}}\right)^{2}-\left(c t-\gamma \frac{v_{0} c}{a_{0}}\right)^{2}}+\frac{c^{2}}{a_{0}} \\
\xi^{2} & =y, \xi^{3}=z, \gamma=\frac{1}{\sqrt{1-\frac{v_{0}^{2}}{c^{2}}}}
\end{aligned}
$$


The speed of light on $x$-axis is

$$
\begin{aligned}
& d y=d \xi^{2}=d z=d \xi^{3}=0, y=\xi^{2}=z=\xi^{3}=0 \\
& c d t=d x \quad, \quad c t=x, \\
& c d \xi^{0}=\frac{d \xi^{1}}{\left(1-\frac{a_{0}}{c^{2}} \xi^{1}\right)}, \\
& c \xi^{0}=-\frac{c^{2}}{a_{0}} \ln \left|1-\frac{a_{0}}{c^{2}} \xi^{1}\right| \rightarrow\left(1-\frac{a_{0}}{c^{2}} \xi^{1}\right)=e^{-\frac{a_{0}}{c} \xi^{0}} \\
& \rightarrow\left(\frac{c^{2}}{a_{0}}-\xi^{1}\right)=\frac{c^{2}}{a_{0}} e^{-\frac{a_{0}}{c} \xi^{0}}
\end{aligned}
$$

Now, if we use the coordinate transformation of the decelerated system given by Eqn. (60) and Eqn. (61), then we have

$$
\begin{aligned}
& c t=\gamma\left(\frac{c^{2}}{a_{0}}-\xi^{1}\right)\left\{\sinh \left(\frac{a_{0} \xi^{0}}{c}\right)-\frac{v_{0}}{c} \cosh \left(\frac{a_{0} \xi^{0}}{c}\right)\right\}+\gamma \frac{v_{0} c}{a_{0}} \\
& =\gamma \frac{c^{2}}{a_{0}} e^{-\frac{a_{0}}{c} \xi^{0}}\left\{\frac{e^{\frac{a_{0}}{c} \xi^{0}}-e^{-\frac{a_{0}}{c} \xi^{0}}}{2}-\frac{v_{0}}{c} \frac{e^{\frac{a_{0}}{c} \xi^{0}}+e^{-\frac{a_{0}}{c} \xi^{0}}}{2}\right\}+\gamma \frac{v_{0} c}{a_{0}} \\
& =\gamma \frac{c^{2}}{a_{0}}\left\{\frac{-e^{-2 \frac{a_{0}}{c} \xi^{0}}+1}{2}-\frac{v_{0}}{c}\left(\frac{e^{-2 \frac{a_{0}}{c} \xi^{0}}+1}{2}-1\right)\right\} \\
& =\gamma \frac{c^{2}}{a_{0}}\left\{\frac{-e^{-2 \frac{a_{0}}{c} \xi^{0}}+1}{2}-\frac{v_{0}}{c} \frac{e^{-2 \frac{a_{0}}{c} \xi^{0}}-1}{2}\right\} \\
& =x=-\gamma\left(\frac{c^{2}}{a_{0}}-\xi^{1}\right)\left\{\cosh \left(\frac{a_{0} \xi^{0}}{c}\right)-\frac{v_{0}}{c} \sinh \left(\frac{a_{0} \xi^{0}}{c}\right)\right\}+\gamma \frac{c^{2}}{a_{0}} \\
& =-\gamma \frac{c^{2}}{a_{0}} e^{-\frac{a_{0}}{c} \xi^{0}}\left\{\frac{e^{\frac{a_{0}}{c} \xi^{0}}+e^{-\frac{a_{0}}{c} \xi^{0}}}{2}-\frac{v_{0}}{c} \frac{e^{\frac{a_{0}}{c} \xi^{0}}-e^{-\frac{a_{0}}{c} \xi^{0}}}{2}\right\}+\gamma \frac{c^{2}}{a_{0}} \\
& =\gamma \frac{c^{2}}{a_{0}}\left\{-\left(\frac{e^{-2 \frac{a_{0}}{c} \xi^{0}}+1}{2}-1\right)+\frac{v_{0}}{c} \frac{-e^{-2 \frac{a_{0}}{c} \xi^{0}}+1}{2}\right\} \\
& =\gamma \frac{c^{2}}{a_{0}}\left\{\frac{-e^{-2 \frac{a_{0}}{c} \xi^{0}}+1}{2}-\frac{v_{0}}{c} \frac{e^{-2 \frac{a_{0}}{c} \xi^{0}}-1}{2}\right\}
\end{aligned}
$$

According to Eqn. (68), the light's Doppler effect of the decelerated system and the inertial system is

$$
\begin{aligned}
& c t=\gamma \frac{c^{2}}{a_{0}}\left\{\frac{-e^{-2 \frac{a_{0}}{c} \xi^{0}}+1}{2}-\frac{v_{0}}{c} \frac{e^{-2 \frac{a_{0}}{c} \xi^{0}}-1}{2}\right\} \\
& =\frac{c^{2}}{a_{0}} \frac{1+\frac{v_{0}}{c}}{\sqrt{1-v_{0}^{2} / c^{2}}} \frac{1-e^{-2 \frac{a_{0}}{c} \xi^{0}}}{2}
\end{aligned}
$$

$$
\begin{aligned}
& c\left(t_{2}-t_{1}\right)=\frac{c}{v}=\frac{c^{2}}{a_{0}} \frac{1+\frac{v_{0}}{c}}{\sqrt{1-v_{0}^{2} / c^{2}}} \frac{-e^{-2 \frac{a_{0}}{c} \xi_{2}^{0}}+e^{-2 \frac{a_{0}}{c} \xi_{1}^{0}}}{2}, \\
& e^{x} \approx 1+x+\frac{x^{2}}{2} \approx \frac{c^{2}}{a_{0}} \frac{\sqrt{1+v_{0} / c}}{\sqrt{1-v_{0} / c}}\left\{\frac{a_{0}}{c}\left(\xi_{2}{ }^{0}-\xi_{1}{ }^{0}\right)\right. \\
& \left.-\frac{a_{0}{ }^{2}}{c^{2}}\left(\xi_{2}{ }^{0}+\xi_{1}{ }^{0}\right)\left(\xi_{2}{ }^{0}-\xi_{1}{ }^{0}\right)\right\} \\
& =\frac{\sqrt{1+v_{0} / c}}{\sqrt{1-v_{0} / c}}\left\{1-\frac{a_{0}}{c}\left(\xi_{1}^{0}+\xi_{2}{ }^{0}\right)\right\} \frac{c}{v_{\xi}} \\
& v \approx \frac{\sqrt{1-v_{0} / c}}{\sqrt{1+v_{0} / c}} \frac{v_{\xi}}{\left\{1-\frac{a_{0}}{c}\left(\xi_{1}^{0}+\xi_{2}^{0}\right)\right\}}>0 \\
& v=\frac{1}{t_{2}-t_{1}}, v_{\xi}=\frac{1}{\xi_{2}^{0}-\xi_{1}^{0}}
\end{aligned}
$$

\section{Conclusion}

We found the Rindler coordinate theory with the initial velocity that uses the tetrad as the new method. The Rindler coordinate theory extended to the Rindler coordinate theory of the accelerated observer that has an initial velocity. This theory treats the slowdown of the system. In fact, the observer with initial velocity does slowdown by the constant negative acceleration in the Rindler's timespace.

We also consider light's Doppler Effect in both the accelerated and decelerated systems. 


\section{References}

[1] S. Weinberg, Gravitation and Cosmology (John Wiley \& Sons Inc., 1972).

[2] W. Rindler, Am. J. Phys. 34, 1174 (1966).

[3] P. Bergman, Introduction to the Theory of Relativity (Dover Pub. Co., Inc., New York, 1976), Ch. V.

[4] C. Misner, K. Thorne and J. Wheeler, Gravitation (W. H. Freedman \& Co., 1973).

[5] S. Hawking and G. Ellis, The Large Scale Structure of Space-Time (Cambridge University Press, 1973).

[6] R. Adler, M. Bazin and M. Schiffer, Introduction to General Relativity (McGrawHill Inc., 1965).

Received: 28 March, 2013

Accepted: 5 July, 2013 